\section{The Agri-Winch: A New Farm Tractor-Mounted Logging Winch ${ }^{1}$}

Over the past few years there has been a widespread interest in farm tractor-mounted winches, especially in eastern Canada. By using these winches, farmers or woodlot owners can supplement their income or secure a source of firewood at a time when their tractor would otherwise be idle. The interest in these types of winches stems from their low purchase price plus the fact that they can be easily mounted on most medium to large-sized farm tractors.

The Agri-Winch is a new type of farm tractor-mounted logging winch. It is constructed to fit any tractor with a power rating of $26 \mathrm{~kW}(35 \mathrm{hp}$ ) and over, and equipped with a threepoint hitch. It's basic design (See Figure S-1) incorporates a combination of sulky, buttplate, winch and fairlead. This transforms a farm tractor into an effective logging machine that resembles a wheeled cable-skidder. The sulky mounting makes it different from other currently available logging winches. FERIC therefore undertook a study to determine if there are advantage over other winches, and to evaluate its productivity.

The Agri-Winch was designed by Lapointe et Frères of Lambton, Quebec. The first unit was built in early 1978. In July 1979, there were 15 units in operation in the Beauce region of southern Quebec. Les Équipements Hardy Inc. of Portneuf Station, Quebec, is presently under contract to manufacture the Agri-Winch for Lapointe et Frères. At the time of writing a series production of 50 units was planned.

The study was conducted in July and November 1979 on three different operations in the Beauce region, Que. The first operation involved a one-day study in a small woodlot using a Case $141060-\mathrm{kW}(80-\mathrm{hp})$ tractor. The softwood component was skidded in tree lengths to a landing 730 metres (2400 feet) away. On Operation 2, softwood sawlogs were skidded over a three day period from a one-hectare clearcut by an Agri-Winch mounted on a Renault 6512-4 48-kW (65-hp) tractor. Operation 3 consisted of two days of timing with the same Renault tractor and operator as on Operation 2. Softwood sawlogs and poplar peeler logs were skidded from a thinning operation to a roadside over a 750 metre (2470 feet) distance.

Results of the FERIC study are presented in Table S-1. Productivity was lower on the second and third operations mainly because $3.8-\mathrm{m}$ log lengths were skidded rather than tree lengths as in Operation 1. Although the skidding distance was much longer in the third operation over the second, the productivity was similar due to the larger load size on the third operation.

Loaded travel speeds averaged $90 \mathrm{~m}(295 \mathrm{ft})$ per minute, $42 \mathrm{~m}(138 \mathrm{ft})$ per minute, and $66 \mathrm{~m}$ (215 ft) per minute, respectively, in the three operations. The main factor governing the travel speds was the skid trail condition.

${ }^{1}$ Further details of this study appear in FERIC Technical Report No. TR-41, entitled "Evaluation of the Agri-Winch: A Farm Tractor-Mounted Logging Winch", by M. Ryans.

\section{L'Agri-Winch: treuil forestier monté sur un tracteur agricole ${ }^{1}$}

Au cours des dernières années, on a constaté, surtout dans l'est du Canada, un intérêt général à l'égard des treuils montés sur des tracteurs agricoles. En utilisant ce genre de treuil, les fermiers ou les propriétaires de lots boisés peuvent ajouter à leur revenu, ou s'assurer une source de bois de chauffage à un moment où leur tracteur demeurerait autrement improductif. L'intérêt suscité provient du faible prix d'achat du treuil, ainsi que de la facilité avec laquelle on peut l'installer sur la plupart des tracteurs agricoles, de dimensions moyennes à grandes.

L'Agri-Winch est un nouveau genre de treuil forestier monté sur un tracteur agricole. Il est construit de façon à convenir à n'importe quel tracteur dont la puissance nominale est de $26 \mathrm{~kW}$ ( $35 \mathrm{hp}$ ) et plus, et muni d'un attelage trois points. Essentiellement, son design (voir figure S-1) est la combinaison d'une arche de débardage sur roues (sulky), d'un bouclier, d'un treuil et d'un guide-câble. On réussit ainsi à transformer un tracteur agricole en un engin d'exploitation efficace qui ressemble à une débardeuse à câbles, montés sur roues. Le fait que l'arche possède des roues en fait un matériel différent des autres treuils forestiers que l'on trouve couramment sur le marché. FERIC a donc entrepris une étude dans le but de déterminer si l'Agri-Winch présente des avantages comparativement aux autres treuils, et d'évaluer sa productivité.

L'Agri-Winch a été conçu par Lapointe et Frères, de Lambton, au Québec. Le premier modèle a été construit au début de 1978 , et en juillet 1979, on en trouvait 15 à l'oeuvre

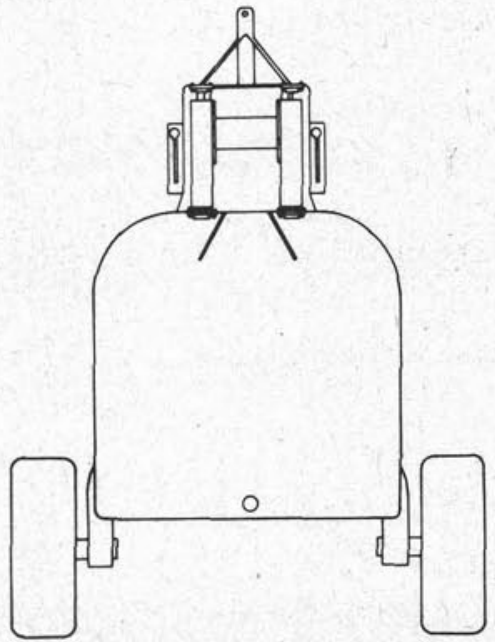

VUE ARRIÈRE

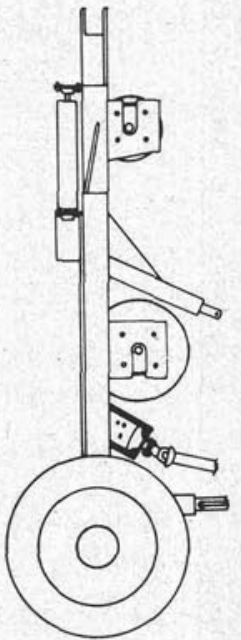

VUE DE CÔTÉ
Figure S-1. Vue arrière et vue de côté de l'Agri-Winch.

\footnotetext{
1 Pour de plus amples renseignements sur cette étude, le rapport technique $\mathrm{N}^{\circ} \mathrm{RT} \cdot 41$, in titulé "Évaluation de l'Agri-Winch: treuil forestier monté sur un tracteur agricole", de M. Ryans est disponible.
} 


\begin{tabular}{|c|c|c|c|}
\hline & Operation 1 & Operation 2 & Operation 3 \\
\hline Tractor Model & Case 1410 & Renault $651-4$ & Renault $651-4$ \\
\hline $\begin{array}{l}\text { Tractor Power, } \\
\text { kilowatts (hp) }\end{array}$ & $60(80)$ & $48(65)$ & $48(65)$ \\
\hline Type of Operation & Selected Cut & Clearcut & Selection Thinning \\
\hline Form of Wood Skidded & Tree Lengths & Sawlogs & Peeler and Sawlogs \\
\hline Travel Distance, $\mathrm{m}(\mathrm{ft})$ & 730 (2395) & $227(745)$ & $753(2470)$ \\
\hline $\begin{array}{l}\text { Volume per Tree or } \\
\qquad \log , m^{3}\left(\mathrm{ft}^{3}\right)\end{array}$ & $0.36(12.7)$ & $0.13(4.7)$ & $0.15(5.4)$ \\
\hline $\begin{array}{l}\text { Volume per Load, } \\
\qquad \mathrm{m}^{3}\left(\mathrm{ft}^{3}\right)\end{array}$ & $3.6(128)$ & $1.4(51)$ & $2.2(78)$ \\
\hline Total Time per Turn, minutes & 27.9 & 32.2 & 52.8 \\
\hline $\begin{array}{l}\text { Productivity, } \\
\mathrm{m}^{3}(\mathrm{ct}) \text { per PHM }\end{array}$ & $7.9(2.8)$ & $2.7(1.0)$ & $2.5(0.9)$ \\
\hline
\end{tabular}

Conventional farm tractor-mounted logging winches that are presently available in Canada have no sulky. They usually contain a mechanical friction plate clutch, a slotted drawbar to attach logs during skidding, and require two adjustable legs to give stability during winching. The main advantages of the Agri-Winch over these presently available winches, made apparent during the study were:

1. Due to the extra axle on the Agri-Winch, there is a better load distribution for skidding. As a result, no weights are required on the front end of the tractor, larger loads are possible, and there is less strain on the 3-point linkages. Better performance when skidding uphill is also possible.

2. With conventional winches, the butt diameter of a log that can be skidded is limited to the clearance from the groundline to the top of the slotted drawbar. The clearance may be less than 60 centimetres ( 24 inches) on some tractors and thus restricts the use or productivity of these winches in stands with large diameter trees. However, the Agri-Winch is able to lift the load much higher because the fairlead is over 1.5 metres ( 5 feet) above the ground. Therefore, the load catches on fewer obstacles and, there is less friction between the ground and the load.

3. If stuck, there is the ability to release the load, drive the dans la région de Québec. Les Équipements Hardy Inc., de Portneuf Station, Québec, ont actuellement le contrat de fabrication du treuil pour le compte de Lapointe et Frères. Au moment où nous écrivons ce rapport, on projette la production en série de 50 treuils.

L'étude eut lieu en juillet et en novembre 1979 dans trois exploitations différentes de la région de la Beauce. La première exploitation comportait une étude d'une journée sur un petit lot boisé où l'on utilisait un tracteur Case 1410 de $60 \mathrm{~kW}(80 \mathrm{hp})$. Les résineux y étaient débardés en troncs entiers jusqu'à une jetée située à 730 mètres (2400 pieds). La seconde exploitation, d'une durée de trois jours, consistait dans le débardage de billes de sciage de résineux, à partir d'une coupe rase d'un hectare, à l'aide de l'AgriWinch monté sur un tracteur Renault $651-4$ de 48 kW (65hp). Quant à la troisième exploitation, c'était une étude chronométrique de deux jours du même tracteur Renault et du même conducteur que l'exploitation 2. On débardait les billes de sciages de résineux et les billes de déroulage de peuplier depuis une opération d'éclaircie jusqu'en bordure de route, sur une distance de 750 mètres (2470 pieds).

Le tableau S-1 donne les résultats de l'étude de FERIC. La productivité plus faible des chantiers 2 et 3 provient surtout du fait qu'on y débardait des billes de 3.8 mètres de long plutôt que des troncs entiers comme au chantier 1. Malgré

\section{Tableau S-1. Sommaire des exploitations}

\begin{tabular}{|c|c|c|c|}
\hline & Chantier 1 & Chantier 2 & Chantier 3 \\
\hline Modèle du tracteur & Case 1410 & Renault $651-4$ & Renault $651-4$ \\
\hline $\begin{array}{l}\text { Puissance du tracteur, } \\
\text { kilowatts (hp) }\end{array}$ & $60(80)$ & $48(65)$ & $48(65)$ \\
\hline Type d'exploitation & $\begin{array}{c}\text { Coupe de jardinage } \\
\text { jardinage }\end{array}$ & Coupe rase & $\begin{array}{l}\text { Éclaircie } \\
\text { jardinatoire }\end{array}$ \\
\hline Forme du bois débardé & Tronc entiers & Billes de sciage & $\begin{array}{l}\text { Billes de sciage } \\
\text { et de déroulage }\end{array}$ \\
\hline Distance de déplacement, $m$ (pi) & $730(2395)$ & $227(745)$ & $753(2470)$ \\
\hline $\begin{array}{l}\text { Volume par arbre ou } \\
\text { par bille, } m^{3}\left(\mathrm{pi}^{3}\right)\end{array}$ & $0.36(12.7)$ & $0.13(4.7)$ & $0.15(5.4)$ \\
\hline $\begin{array}{l}\text { Volume par charge, } \\
\mathrm{m}^{3}\left(\mathrm{pi}^{3}\right)\end{array}$ & $3.6(128)$ & $1.4(51)$ & $2.2(78)$ \\
\hline Temps total par cycle, minutes & 27.9 & 32.2 & 52.8 \\
\hline $\begin{array}{l}\text { Productivité, } \\
\mathrm{m}^{3} \text { (cu) par HMP }\end{array}$ & $7.9(2.8)$ & $2.7(1.0)$ & $2.5(0.9)$ \\
\hline
\end{tabular}




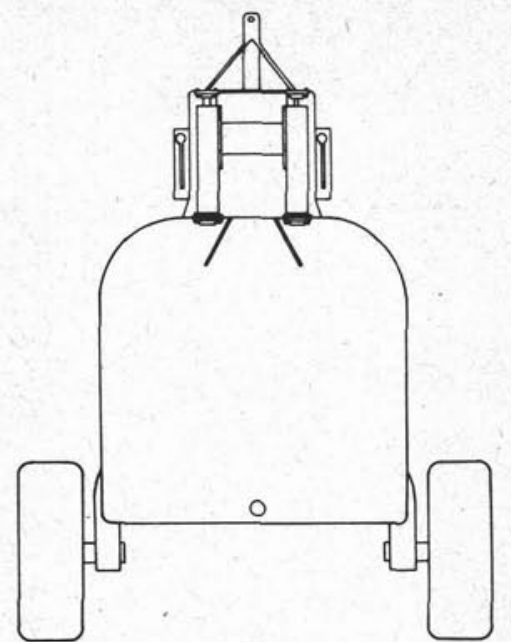

BACK VIEW

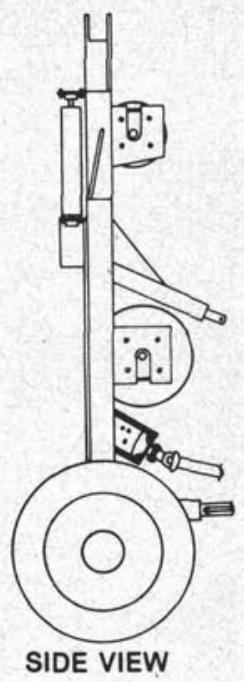

Figure S-1. Back and side views of the Agri-Winch

factor forward out of the wet area, and then winch on the load. (This is not possible where the individual trees are hooked onto a slotted drawbar).

4. The fairlead and buttplate design gives greater flexibility in loading technique over the slotted drawbar found on many conventional farm tractor-mounted logging winches.

5. The manual clutch requires no adjustment, and is simple, rugged construction requires few repairs. Clutch adjustment has been reported to be a problem on some other winches.

6. The Agri-Winch has improved turning ability, when compared to farm tractor-mounted winches having loads attached to the slotted drawbar.

Some disadvantages of the Agri-Winch over the presently available winches were:

1. The Agri-Winch with tractor is a longer unit, and, in a confined area may be more difficult to manoeuver. However, since the Agri-Winch can be raised (to avoid obstacles) about $0.5 \mathrm{~m}(1.6 \mathrm{ft})$ above the ground using the 3-point hydraulic hitch this is a minor problem.

2. The larger size of the Agri-Winch may sometimes impede the operator's visibility to the rear of the tractor. une distance de débardage supérieure dans le troisième chantier comparativement au second, on obtint une productivité semblable qui s'explique par des charges de plus fortes dimensions dans le troisième chantier.

Les vitesses de déplacement en charge atteignaient en moyenne $90 \mathrm{~m}$ (295 pi) par minute, $42 \mathrm{~m}$ (138 pi) par minute, et $66 \mathrm{~m}$ (215 pi) par minute, respectivement. Le principal facteur qui déterminait la vitesse de déplacement était l'état du sentier de débardage.

Les treuils forestiers traditionnels, montés sur des tracteurs agricoles, que l'on trouve actuellement au Canada, n'ont pas d'arche de débardage sur roues. Ils comportent généralemnt un embrayage mécanique à plateau, une barre d'attelage à encoches pour attacher les billes lors du débardage, et ils demandent deux béquilles réglables qui assurent la stabilité durant le tirage. Comparativement à ces treuils actuellement sur le marché, l'Agri-Winch possède plusieurs avantages que l'étude a fait ressortir et dont les principaux sont les suivants:

1. Étant donné l'essieu traditionnel de l'Agri-Winch, on obtient une meilleure distribution de la charge pour le débardage. On n'a donc pas besoin d'ajouter de poids à l'avant du tracteur, il est possible de transporter des charges plus fortes et la tension exercée sur l'attelage trois point est plus faible. On obtient également un meilleur rendement lorsqu'on débarde en montant.

2. Avec les treuils traditionnels, on ne peut pas débarder de billes dont le gros bout a un diamètre supérieur à l'espace compris entre le niveau du sol et le sommet de la base d'attelage à encoches. Chez certains tracteurs, cet espace est parfois inférieur à 60 centimètres $(24$ pouces), ce qui limite l'utilisation ou la productivité de ces treuils dans des peuplements où les arbres sont de plus fort diamètre. L'Agri-Winch, au contraire, peut lever la charge beaucoup plus haut puisque le guidecâble atteint plus de 1.5 mètres ( 5 pieds) au-dessus du sol. La charge risque donc d'accrocher à un moins grand nombre d'obstacles et il s'exerce moins de friction entre le sol et la charge.

3. Si le tracteur reste pris, il est toujours possible de relâcher la charge, de conduire le tracteur hors du terrain humide, puis de tirer la charge à l'aide du treuil. (Ceci est impossible lorsque les arbres sont accrochés un à un dans une barre d'attelage à encoches.)

4. Le design du guide-câble et du bouclier donne plus de souplesse quant à la technique de chargement à encoches que présentent plusieurs treuils traditionnels montés sur des tracteurs agricoles.

5. L'embrayage manuel ne demande pas de réglage, et sa construction simple et robuste ne donne lieu qu'à peu de réparations. Le réglage de l'embrayage semble constituer un problème dans le cas de plusieurs autres treuils.

6. L'Agri-Winch est doté d'une facilité de braquage que ne possèdent pas les treuils dont la charge est attachée à la barre d'attelage à encoches.

Voici quelques-uns des inconvénients que présente l'AgriWinch, comparativement aux treuils actuellement sur le marché:

1. L'Agri-Winch et le tracteur forment un ensemble plus long, qui sera plus difficile à manoeuvrer, si l'espace est restreint. Cependant, comme on peut élever l'AgriWinch (pour éviter les obstacles) d'environ $0.5 \mathrm{~m}$ (1.6 pi) au-dessus du sol à l'aide de l'attelage hydraulique trois points, ce n'est là qu'un problème mineur.

2. Les plus grandes dimensions de l'Agri-Winch peuvent parfois gêner la visibilité du conducteur vers l'arrière du tracteur. 